Enferm Bras 2019;18(4):518-27

https://doi.org/10.33233/eb.v18i4.2690

\title{
ARTIGO ORIGINAL \\ Opiniões de universitários acerca da experiência da primeira exposição ao álcool e outras drogas
}

Daniel Augusto da Silva*, Carlos Fabiano Munir Gomes**, Josiane Viana Cardoso**, Ronaldo José Pereira Junior**, Rosângela Gonçalves da Silva***

*Enfermeiro, Doutorando em Ciências (EPE/UNIFESP), Fundação Educacional do Município de Assis (FEMA), ${ }^{* *}$ Enfermeiro, Fundação Educacional do Município de Assis (FEMA), ${ }^{\star * *}$ Enfermeira, Doutoranda em Biociências (UNESP), Fundação Educacional do Município de Assis (FEMA)

Recebido em 5 de dezembro de 2018; aceito em 2 de julho de 2019.

Correspondência: Daniel Augusto da Silva, Avenida Getúlio Vargas, 1200, Vila Nova Santana, 19807-130 Assis SP

Daniel Augusto da Silva: daniel.augusto@unifesp.br

Carlos Fabiano Munir Gomes: carlos.fabiano93@gmail.com

Josiane Viana Cardoso: josiane.jv11@gmail.com

Ronaldo José Pereira Junior: ronaldopereirajunior@hotmail.com

Rosângela Gonçalves da Silva: roseziquinelli@gmail.com

\section{Resumo}

Objetivo: Conhecer as opiniões de estudantes universitários sobre experimentar os diferentes tipos de drogas existentes, compreendendo as drogas lícitas e as ilícitas. Métodos: Trata-se de pesquisa transversal, de abordagem quantitativa, realizada com 406 estudantes universitários, no $3^{\text {o }}$ trimestre de 2017, com questionários elaborados pelos autores. Os dados foram analisados por meio de análise estatística descritiva. Resultados: Os índices gerais de aprovação para experimentação e o tipo de drogas são: 29,8\% para tabaco, 68,2\% para álcool, 15,5\% para maconha, 3,7\% para crack, $4,7 \%$ para anfetaminas, $4,7 \%$ para inalantes, $13,1 \%$ para hipnóticos, 6,4\% para alucinógenos, e 6,4\% para opiáceos, o que traduz um comportamento de admissão da possibilidade de experimentação de todos os tipos de substâncias pelos participantes da pesquisa. Conclusão: A necessidade de investimento em políticas educacionais relacionadas à prevenção do primeiro contato com essas substâncias é clara, quando considerado o cenário real de contato com essas substâncias na população universitária.

Palavras-chave: estudantes, comportamento de procura de droga, transtornos relacionados ao uso de substâncias.

\section{Abstract \\ University students' opinions on their first exposure to alcohol and other drugs}

Aim: To know the opinions of university students about experimenting with different types of drugs, including licit and illicit drugs. Methods: This is a cross-sectional, quantitative approach, carried out with 406 university students, in the 3rd quarter of 2017, with questionnaires prepared by the authors. Data were analyzed through descriptive statistical analysis. Results: The general approval ratings for experimentation and the type of drugs are: $29.8 \%$ for tobacco, $68.2 \%$ for alcohol, $15.5 \%$ for marijuana, $3.7 \%$ for crack cocaine, $4.7 \%$ for amphetamines, $4.7 \%$ for inhalants, $13.1 \%$ for hypnotics, $6.4 \%$ for hallucinogens, and $6.4 \%$ for opiates, which translates into an admission behavior of the possibility of experimentation of all types of substances by participants in the search. Conclusion: The need to invest in educational policies related to the prevention of first contact with these substances is clear when considering the real scenario of contact with these substances in the university population.

Key-words: students, drug-seeking behavior, substance-related disorders.

\section{Resumen}




\section{Opiniones de universitarios sobre la experiencia de la primera exposición al alcohol y otras drogas}

Objetivo: Conocer las opiniones de estudiantes universitarios sobre experimentar los diferentes tipos de drogas, incluidas las drogas lícitas e ilícitas. Métodos: Se trata de una investigación transversal, de abordaje cuantitativo, realizada con 406 estudiantes universitarios, en 2017, con cuestionarios elaborados por los autores. Los datos fueron analizados con análisis estadístico descriptivo. Resultados: Los índices generales de aprobación para experimentación y el tipo de drogas son: $29,8 \%$ para tabaco, $68,2 \%$ para alcohol, $15,5 \%$ para marihuana, 3,7\% para crack, 4,7\% para anfetaminas, 4,7\% para los inhalantes, $13,1 \%$ para los hipnóticos, $6,4 \%$ para los alucinógenos y $6,4 \%$ para los opiáceos, lo que refleja un comportamiento de admisión de la posibilidad de experimentación de todo tipo de sustancias por los participantes de la encuesta. Conclusión: La necesidad de inversión en políticas educativas relacionadas con la prevención del primer contacto con esas sustancias es clara, cuando se considera el escenario real de contacto con esas sustancias en la población universitaria.

Palabras-clave: estudiantes, comportamiento de búsqueda de drogas, trastornos relacionados con sustancias.

\section{Introdução}

A questão do uso e dependência do álcool e outras substâncias psicoativas têm correspondido a um problema cada vez mais presente e frequente, em todas as sociedades, em nível mundial, fato que tem se traduzido em grave problema social e de saúde pública. Os usuários que outrora eram considerados marginais em um contexto social estão passando a ser identificados como membros da família, amigos, vizinhos, colegas de trabalho ou pessoas que possuam qualquer outro laço de convivência $[1,2]$.

$\mathrm{Na}$ análise do envolvimento com álcool e outras substâncias na população geral, é observável que os estudantes universitários apresentam um consumo de drogas mais intenso e frequente, quando comparados a outras parcelas da população [3].

Com essa situação, é evidente a necessidade do conhecimento sobre o envolvimento dos estudantes com as drogas, pois as atividades de prevenção têm mais resultados nessa faixa etária, e é nessa faixa que ocorre o primeiro contato com as drogas para a maioria das pessoas, além de que o acesso a uma variedade de substâncias, que tem sido mais facilitado a essa população, que vivencia uma série de impactos negativos para a saúde por conta do precoce contato com essas substâncias [3].

Por conta disso, várias pesquisas locais, regionais e nacionais têm sido realizadas, para rastreamento do envolvimento com álcool e outras substâncias entre a população universitária, possibilitando até a análise anual do comportamento em relação às drogas, com a oportunidade de conhecimento sobre a diminuição, manutenção ou aumento do índice de envolvimento com drogas nesta parcela da população [3].

O envolvimento com o álcool e outras substâncias pode ser classificado por estágios, e estes compreendem a experimentação, que marca o início do contato com a droga; o uso social ou ocasional, que se refere ao uso da droga com frequência irregular e em quantidades modestas; o uso regular, que caracteriza um padrão de uso frequente; o uso situacional, quando o consumo é associado a um objeto específico; o uso compulsivo, caracterizado por períodos de uso de grandes quantidades intercalados por períodos de abstinência; o abuso, que é o uso da droga com apresentação de problemas significativos associados a ela, e a dependência, quando ocorre a presença de sintomas cognitivos, comportamentais e fisiológicos que indicam a continuação do uso de substâncias apesar dos problemas relacionados a ela [4].

O estágio de experimentação, com a ocorrência do primeiro contato com as drogas, é apontado na literatura como maior facilidade e vulnerabilidade na adolescência, em decorrência do impacto das transformações físicas e psíquicas características desta faixa etária [5].

A partir do expressivo número de pessoas que apresentam envolvimento com álcool e outras drogas, esta pesquisa tem como objetivo conhecer as opiniões de estudantes universitários sobre experimentar os diferentes tipos de drogas existentes, compreendendo as drogas lícitas e as ilícitas.

Com os dados obtidos por meio da avaliação dos estudantes universitários, haverá possibilidade de contribuição na compreensão e planejamento de estratégias de prevenção e recuperação relacionadas à ocorrência do envolvimento com álcool, tabaco e outras substâncias nesta população, tendo em vista a vulnerabilidade a qual estão expostos, e os altos índices de utilização, discutidos amplamente na literatura. 
Trata-se de uma pesquisa de levantamento, exploratória, descritiva, de abordagem quantitativa.

O estudo foi realizado em uma instituição de ensino superior localizada em cidade do centro-oeste do estado de São Paulo, Brasil. A população de estudantes universitários regularmente matriculados na instituição de ensino em 2017 era de 2.164, e a amostra calculada com grau de confiança de $95 \%$, e margem de erro de $5 \%$ foi de 326 participantes. Participaram 406 estudantes universitários, que compuseram a amostra final.

Optou-se por um modelo de amostragem probabilística aleatória estratificada proporcional, para que houvesse representantes dos cursos de graduação oferecidos pela instituição, a saber: Administração, Análise de Sistemas, Ciências da Computação, Ciências Contábeis, Direito, Enfermagem, Fotografia, Medicina, Publicidade e Propaganda e Química.

Todos os participantes da pesquisa assinaram o Termo de Consentimento Livre e Esclarecido, concordando a participação e, após, responderam os instrumentos respectivos a esta pesquisa, de acordo com a legislação específica para pesquisas com seres humanos [6].

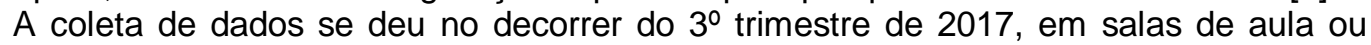
espaços internos da instituição, que proporcionassem privacidade para o desenvolvimento da mesma. Houve a aplicação de questionário semiestruturado, elaborado pelos autores, para identificação de dados sociodemográficos e instrumento acerca da aprovação ou desaprovação da experimentação de álcool e outras substâncias, ambos os instrumentos elaborados pelos autores. Os dados foram analisados com uso de análise estatística descritiva e de acordo com as instruções para aplicação do instrumento selecionado. Também foi realizado o teste de QuiQuadrado de Pearson, no Software SPSS versão 20.0, para verificar as diferenças existentes entre o sexo e o envolvimento com substâncias. A pesquisa foi submetida e aprovada pelo Comitê de Ética em Pesquisa do Hospital Amaral Carvalho, sob parecer número 2.105.041, de 07 de junho de 2017.

\section{Resultados}

Obtiveram-se, neste estudo, respostas afirmativas sobre a aprovação de experimentação de todas as substâncias, conforme descrito na Tabela I.

Tabela I - Índice geral de aprovação de experimentação do álcool e outras substâncias por estudantes universitários, Assis, São Paulo, Brasil, $2019(n=406)$.

\begin{tabular}{ll}
\hline Substância & Índice de aprovação $\mathbf{n}(\%)$ \\
\hline Derivados do tabaco & $121(29,8)$ \\
Bebidas alcóolicas & $277(68,2)$ \\
Maconha & $63(15,5)$ \\
Crack & $15(3,7)$ \\
Anfetamina & $19(4,7)$ \\
Inalante & $19(4,7)$ \\
Hipnótico & $53(13,1)$ \\
Alucinógeno & $26(6,4)$ \\
Opiáceo & $26(6,4)$ \\
\hline
\end{tabular}

Quanto à participação, este estudo contou com a participação voluntária de 406 estudantes universitários, dos quais $240(59,1 \%)$ eram do sexo feminino e $166(40,9 \%)$ do sexo masculino. Demais dados a respeito da caracterização dos participantes e a aprovação da experimentação do álcool, tabaco e outras drogas estão descritos na Tabela II.

Tabela II - Aprovação da experimentação do álcool ou outras substâncias por estudantes universitários e as diferenças de comportamento em relação às variáveis sociodemográficas, Assis, São Paulo, Brasil, 2019 ( $n=406)$. Teste de Qui-quadrado de Pearson ou Exato de Fisher. Variável Tabaco Álcool Maconha Crack Anfetamina Inalante Hipnótico Alucinógeno Opiáceo 


\begin{tabular}{|c|c|c|c|c|c|c|c|c|c|}
\hline n (\%) & n (\%) & n (\%) & n (\%) & n (\%) & n (\%) & n (\%) & n (\%) & n (\%) & n (\%) \\
\hline Sexo & & & & & & & & & \\
\hline $\begin{array}{l}\text { Feminino } \\
240(59,1)\end{array}$ & $\begin{array}{r}63 \\
(26,2)\end{array}$ & $\begin{array}{r}164 \\
(68,3)\end{array}$ & $\begin{array}{r}36 \\
(15,0)\end{array}$ & $\begin{array}{r}6 \\
(2,5)\end{array}$ & $\begin{array}{r}8 \\
(3,3)\end{array}$ & $\begin{array}{r}9 \\
(3,8)\end{array}$ & $\begin{array}{r}41 \\
(17,1)\end{array}$ & $\begin{array}{r}11 \\
(4,6)\end{array}$ & $\begin{array}{r}16 \\
(6,7)\end{array}$ \\
\hline Masculino & 58 & 113 & 27 & 9 & 11 & 10 & 12 & 15 & 10 \\
\hline $166(40,9)$ & $(34,9)$ & $(68,1)$ & $(16,3)$ & $(5,4)$ & $(6,6)$ & $(6,0)$ & $(7,2)$ & $(9,0)$ & $(6,0)$ \\
\hline Valor-p & 0,060 & 0,956 & 0,729 & 0,179 & 0,152 & 0,341 & 0,004 & 0,098 & 0,840 \\
\hline \multicolumn{10}{|l|}{ Faixa etária } \\
\hline 17 a 26 anos & 105 & 236 & 52 & 13 & 15 & 16 & 41 & 22 & 20 \\
\hline $333(82,0)$ & $(31,5)$ & $(70,9)$ & $(15,6)$ & $(3,9)$ & $(4,5)$ & $(4,8)$ & $(12,3)$ & $(6,6)$ & $(6,0)$ \\
\hline 27 a 36 anos & & 31 & 7 & 2 & 4 & 3 & 9 & 4 & 6 \\
\hline $46(11,3)$ & $(26,1)$ & $(67,4)$ & $(15,2)$ & $(4,3)$ & $(8,7)$ & $(6,5)$ & $(19,6)$ & $(8,7)$ & $(13,0)$ \\
\hline 37 a 46 anos & & & 2 & 0 & 0 & 0 & 1 & 0 & 0 \\
\hline $12(3,0)$ & $(8,3)$ & $(41,7)$ & $(16,7)$ & $(0,0)$ & $(0,0)$ & $(0,0)$ & $(8,3)$ & $(0,0)$ & $(0,0)$ \\
\hline 47 a 58 anos & & & 2 & 0 & 0 & 0 & 2 & 0 & 0 \\
\hline $15(3,7)$ & $(20,0)$ & $(33,3)$ & $(13,3)$ & $(0,0)$ & $(0,0)$ & $(0,0)$ & $(13,3)$ & $(0,0)$ & $(0,0)$ \\
\hline Valor-p & 0,250 & 0,005 & 1,000 & 0,897 & 0,456 & 0,866 & 0,547 & 0,675 & 0,242 \\
\hline \multicolumn{10}{|l|}{$\begin{array}{l}\text { Orientação } \\
\text { sexual }\end{array}$} \\
\hline $\begin{array}{l}\text { Heterossexual } \\
380(93,6)\end{array}$ & $\begin{array}{r}110 \\
(28,9)\end{array}$ & $\begin{array}{r}259 \\
(68,2)\end{array}$ & $\begin{array}{r}52 \\
(13,7)\end{array}$ & $\begin{array}{r}14 \\
(3,7)\end{array}$ & $\begin{array}{r}17 \\
(4,5)\end{array}$ & $\begin{array}{r}18 \\
(4,7)\end{array}$ & $\begin{array}{r}51 \\
(13,4)\end{array}$ & $\begin{array}{r}20 \\
(5,3)\end{array}$ & $\begin{array}{r}25 \\
(6,6)\end{array}$ \\
\hline Homossexual & & 10 & & 1 & 1 & 1 & & 2 & 1 \\
\hline $15(3,7)$ & $(60,0)$ & $(66,7)$ & $(40,0)$ & $(6,7)$ & $(6,7)$ & $(6,7)$ & $(13,3)$ & $(13,3)$ & $(6,7)$ \\
\hline Bissexual & & & 5 & 0 & 1 & 0 & 0 & 4 & 0 \\
\hline $11(2,7)$ & $(18,2)$ & $(72,7)$ & $(45,5)$ & $(0,0)$ & $(9,1)$ & $(0,0)$ & $(0,0)$ & $(36,4)$ & $(0,0)$ \\
\hline Valor-p & 0,032 & 1,000 & 0,002 & 0,637 & 0,350 & 0,718 & 0,575 & 0,002 & 1,000 \\
\hline \multicolumn{10}{|l|}{ Cor de pele } \\
\hline Branca & 108 & 227 & 48 & 15 & 17 & 17 & 46 & 20 & 23 \\
\hline $330(81,3)$ & $(32,7)$ & $(68,8)$ & $(14,5)$ & $(4,5)$ & $(5,2)$ & $(5,2)$ & $(13,9)$ & $(6,1)$ & $(7,0)$ \\
\hline Parda & & 40 & & 0 & 2 & 2 & 6 & 6 & 3 \\
\hline $59(14,5)$ & $(15,3)$ & $(67,8)$ & $(18,6)$ & $(0,0)$ & $(3,4)$ & $(3,4)$ & $(10,2)$ & $(10,2)$ & $(5,1)$ \\
\hline Preta & & 10 & 4 & 0 & 0 & 0 & 1 & 0 & 0 \\
\hline $14(3,4)$ & $(28,6)$ & $(71,4)$ & $(28,6)$ & $(0,0)$ & $(0,0)$ & $(0,0)$ & $(7,1)$ & $(0,0)$ & $(0,0)$ \\
\hline Amarela & 0 & 0 & 0 & 0 & 0 & 0 & 0 & 0 & 0 \\
\hline $2(0,5)$ & $(0,0)$ & $(0,0)$ & $(0,0)$ & $(0,0)$ & $(0,0)$ & $(0,0)$ & $(0,0)$ & $(0,0)$ & $(0,0)$ \\
\hline Indígena & 0 & 0 & 0 & 0 & 0 & 0 & 0 & 0 & 0 \\
\hline $1(0,2)$ & $(0,0)$ & $(0,0)$ & $(0,0)$ & $(0,0)$ & $(0,0)$ & $(0,0)$ & $(0,0)$ & $(0,0)$ & $(0,0)$ \\
\hline Valor-p & 0,044 & 0,169 & 0,453 & 0,328 & 0,886 & 0,886 & 0,789 & 0,519 & 0,791 \\
\hline \multicolumn{10}{|l|}{ Estado civil } \\
\hline Solteiro & 114 & 253 & 56 & 13 & 18 & 18 & 48 & 25 & 25 \\
\hline $358(88,2)$ & $(31,8)$ & $(70,7)$ & $(15,6)$ & $(3,6)$ & $(5,0)$ & $(5,0)$ & $(13,4)$ & $(7,0)$ & $(7,0)$ \\
\hline Casado & & 13 & 3 & 1 & 1 & 1 & 3 & 1 & 1 \\
\hline $27(6,7)$ & $(7,4)$ & $(48,1)$ & $(11,1)$ & $(3,7)$ & $(3,7)$ & $(3,7)$ & $(11,1)$ & $(3,7)$ & $(3,7)$ \\
\hline União estável & & & & 1 & 0 & 0 & 1 & 0 & 0 \\
\hline $14(3,4)$ & $(35,7)$ & $(57,1)$ & $(28,6)$ & $(7,1)$ & $(0,0)$ & $(0,0)$ & $(7,1)$ & $(0,0)$ & $(0,0)$ \\
\hline Divorciado & 0 & & 0 & 0 & 0 & 0 & 1 & 0 & 0 \\
\hline $6(1,5)$ & $(0,0)$ & $(50,0)$ & $(0,0)$ & $(0,0)$ & $(0,0)$ & $(0,0)$ & $(16,7)$ & $(0,0)$ & $(0,0)$ \\
\hline Viúvo & 0 & 0 & 0 & 0 & 0 & 0 & 0 & 0 & 0 \\
\hline $1(0,2)$ & $(0,0)$ & $(0,0)$ & $(0,0)$ & $(0,0)$ & $(0,0)$ & $(0,0)$ & $(0,0)$ & $(0,0)$ & $(0,0)$ \\
\hline Valor-p & 0,014 & 0,028 & 0,504 & 0,688 & 1,000 & 1,000 & 0,900 & 0,927 & 0,927 \\
\hline
\end{tabular}

Sobre esses dados, estatisticamente é possível afirmar que a aprovação da experimentação de hipnóticos está associada ao sexo, a experimentação do álcool está associada a faixa etária, a experimentação do tabaco, da maconha e de alucinógenos estão associadas à orientação sexual, a experimentação do tabaco está associada à cor de pele, e a aprovação da experimentação do tabaco e do álcool estão associadas ao estado civil.

Quanto as variáveis acadêmicas, há diferença estatística significante para quase a totalidade dos tipos de drogas pesquisados, quando analisada a diferença de comportamento para a aprovação em relação ao curso de graduação no qual o estudante está vinculado, possibilitando a afirmação de que há diferença de comportamento para aprovação de diferentes tipos de drogas (Tabela III).

Tabela III - Aprovação da experimentação do álcool ou outras substâncias por estudantes universitários e as diferenças de comportamento em relação às variáveis acadêmicas, Assis, São Paulo, Brasil, 2019 ( $n=406)$.

\begin{tabular}{|c|c|c|c|c|c|c|c|c|c|}
\hline $\begin{array}{l}\text { Variável } \\
\text { n (\%) }\end{array}$ & $\begin{array}{l}\text { Tabaco } \\
\text { n (\%) }\end{array}$ & $\begin{array}{l}\text { Álcool } \\
\mathrm{n}(\%)\end{array}$ & $\begin{array}{l}\text { Maconha } \\
\text { n (\%) }\end{array}$ & $\begin{array}{l}\text { Crack } \\
\text { n }(\%)\end{array}$ & $\begin{array}{l}\text { Anfetamina } \\
\mathrm{n}(\%)\end{array}$ & $\begin{array}{l}\text { Inalante } \\
\mathrm{n}(\%)\end{array}$ & $\begin{array}{l}\text { Hipnótico } \\
\text { n (\%) }\end{array}$ & $\begin{array}{l}\text { Alucinógeno } \\
\mathbf{n}(\%)\end{array}$ & $\begin{array}{l}\text { Opiáceo } \\
\text { n (\%) }\end{array}$ \\
\hline
\end{tabular}




\begin{tabular}{lrrrrrrrrr} 
Administração & 29 & 28 & 5 & 2 & 2 & 2 & 1 & 1 & 1 \\
$45(11,1)$ & $(64,4)$ & $(62,2)$ & $(11,1)$ & $(4,4)$ & $(4,4)$ & $(4,4)$ & $(2,2)$ & $(2,2)$ & $(2,2)$ \\
A sistemas & 14 & 21 & 0 & 0 & 0 & 0 & 0 & 0 & 0 \\
$35(8,6)$ & $(40,0)$ & $(60,0)$ & $(0,0)$ & $(0,0)$ & $(0,0)$ & $(0,0)$ & $(0,0)$ & $(0,0)$ & $(0,0)$ \\
C & 13 & 36 & 0 & 0 & 0 & 0 & 0 & 0 & 0 \\
Computação & $(36,1)$ & $(100,0)$ & $(0,0)$ & $(0,0)$ & $(0,0)$ & $(0,0)$ & $(0,0)$ & $(0,0)$ & $(0,0)$ \\
$36(8,9)$ & & & & & & & & 13 & 12 \\
Direito & 34 & 103 & 31 & 4 & & & & \\
170 (41,9) & $(20,0)$ & $(60,6)$ & $(18,2)$ & $(2,4)$ & $(4,7)$ & $(2,9)$ & $(17,1)$ & $(7,6)$ & $(7,1)$ \\
Enfermagem & 8 & 23 & 2 & 0 & 1 & 0 & 8 & 0 & 5 \\
$33(8,1)$ & $(24,2)$ & $(69,7)$ & $(6,1)$ & $(0,0)$ & $(3,0)$ & $(0,0)$ & $(24,2)$ & $(0,0)$ & $(15,2)$ \\
Fotografia & 5 & 10 & 7 & 0 & 0 & 3 & 2 & 3 & 0 \\
10 (2,5) & $(50,0)$ & $(100,0)$ & $(70,0)$ & $(0,0)$ & $(0,0)$ & $(30,0)$ & $(20,0)$ & $(30,0)$ & $(0,0)$ \\
Medicina & 7 & 18 & 6 & 4 & 3 & 3 & 5 & 4 & 3 \\
23 (5,7) & $(30,4)$ & $(78,3)$ & $(26,1)$ & $(17,4)$ & $(13,0)$ & $(13,0)$ & $(21,7)$ & $(17,4)$ & $(13,0)$ \\
P Propaganda & 8 & 20 & 8 & 3 & 3 & 4 & 4 & 3 & 3 \\
28 (6,9) & $(28,6)$ & $(71,4)$ & $(28,6)$ & $(10,7)$ & $(10,7)$ & $(14,3)$ & $(14,3)$ & $(10,7)$ & $(10,7)$ \\
Química & 3 & 18 & 4 & 2 & 2 & 2 & 4 & 2 & 2 \\
26 (6,7) & $(11,5)$ & $(69,2)$ & $(15,4)$ & $(7,7)$ & $(7,7)$ & $(7,7)$ & $(15,4)$ & $(7,7)$ & $(7,7)$ \\
Valor-p & $<0,001$ & $<0,001$ & $<0,001$ & 0,011 & 0,211 & 0,001 & $<0,001$ & 0,003 & 0,064 \\
\hline
\end{tabular}

Discussão

O início do uso das drogas é observado por diferentes razões, que incluem o julgamento pessoal do indivíduo sobre o prazer da experiencia em si, o efeito estimulado pelas drogas sobre as pressões cotidianas, podendo, até este motivo para uso, ser devido ao desejo de alcance de um estado religioso ou espiritual [7].

Vários fatores podem estar intrínsecos ao comportamento do uso das drogas, como o conhecimento sem censura sobre o uso de drogas por celebridades, que por vezes são consideradas ídolos, e podem induzir a imitação do comportamento pessoal, a facilidade de acesso às drogas, sejam elas lícitas ou ilícitas, a pressão social, e fatores genéticos que parecem sugerir maior susceptibilidade de pessoas às drogas [8].

Dessa forma, muitas pessoas, durante a vida, experimentam uma variedade de substâncias que são causadoras de dependência, mas não se pode afirmar que todas as pessoas que experimentam essas substâncias serão dependentes delas, para que esse comportamento se efetive, uma complexa interação de fatores psicológicos, neurobiológicos e individuais precisa ocorrer [9].

Este fato nos permite afirmar que, dependendo do contexto, cada substância pode influenciar o comportamento do indivíduo de forma inofensiva, com poucos riscos, ou pode acarretar em prejuízos biológicos, psicológicos e sociais, sendo observado que as situações de risco como os problemas com a polícia e a justiça, a exposição e envolvimento com diversos tipos de violência, a vivência de comportamento sexual de risco e a possibilidade de desenvolver a dependência química serão proporcionais ao maior tempo de exposição a elas, considerando o início de consumo mais precoce [10].

Em universitários, o envolvimento com álcool e outras drogas pode estar relacionada à intensa mudança de hábitos, consequência de realidades cognitivas e emocionais inesperadas, como o afastamento da família, adaptação ao novo estilo de vida e ao ambiente desconhecido, novas amizades, novos desafios e preocupações, fatos que o tornam susceptíveis a adoção de hábitos não saudáveis, como o envolvimento a essas substâncias [11-13].

Neste estudo, obteve-se aprovação para experimentação para todas as drogas, no entanto as características sociodemográficas, diferenças individuais, revelaram diferenças nas opiniões e condutas quanto a esse comportamento.

A aprovação para experimentação do álcool foi a de maior incidência, mesmo sendo de conhecimento que o álcool e o tabaco são as substâncias que mais matam em todo o mundo [14].

A situação da alta incidência de experimentação do álcool é fato que se semelha a outras pesquisas realizadas com essa temática, não somente no Brasil, mas abrangendo estudantes portugueses $(59,0 \%)$ [15-20].

A análise de comportamento relacionado à experimentação de álcool e outras substâncias, em relação ao sexo, revelou, na população feminina, a maior aprovação para experimentação do álcool (feminino 68,3\%, masculino 68,1\%), de hipnóticos (feminino 17,1\%, masculino 7,2\%), e de opiáceos (feminino 6,7\%, masculino 6,0\%). 
O comportamento da população feminina em relação ao uso de substâncias psicoativas tem apresentado aumento gradual nos últimos anos, com o aumento e dependência do álcool, e prevalência do uso das substâncias estimulantes, cuja utilização é duas ou três vezes maior quando comparado o uso na população masculina [21].

A questão do uso de álcool, tabaco e medicamentos na população feminina tem sido prevalente, entretanto, mesmo que pouco aceito socialmente, o envolvimento e uso de substâncias ilícitas pelas mulheres tem revelado importante mudança de comportamento, no que se refere a maior aprovação para experimentação de drogas ilícitas [22]. Esses fatos puderam ser comprovados neste estudo, no qual houve envolvimento desta população para todos os tipos de substâncias.

A questão dos altos índices de utilização do álcool está relacionada à permissão desta ação por parte da sociedade e do governo, todavia, deve-se ressaltar que a grande maioria das pessoas que faz uso de álcool não o faz de modo problemático, contudo, devem estar incluídas nas atividades de educação e prevenção de riscos, danos e vulnerabilidades [23-24].

Quanto ao estado civil, a aprovação para experimentação do álcool esteve presente de forma significativa, chegando ao índice de aprovação em $68,2 \%$ para os solteiros.

O fator estado civil e a interferência no uso de substâncias possuem ampla discussão na literatura, com a afirmação de que a ausência de um parceiro pode resultar como risco para o envolvimento com estas substâncias, uma vez que o parceiro pode se constituir como auxilio psicológico frente aos acontecimentos estressantes, culminando na não recorrência às drogas [25-27].

A orientação sexual revelou diferenças importantes de comportamento quanto à experimentação de substâncias. Tabaco, álcool, maconha, anfetaminas, alucinógenos obtiveram aprovação para todas as populações. A aprovação da experimentação do tabaco foi maior para a população homossexual $(50,0 \%)$, assim como o crack $(5,6 \%)$ e inalantes $(5,6 \%)$. A população heterossexual demonstrou maior índice de aprovação para experimentação de hipnóticos $(13,4 \%)$ e opiáceos $(6,6 \%)$. A população bissexual apresentou comportamento de total desaprovação para experimentação de crack, inalantes, hipnóticos e opiáceos, todavia, foi a população de maior aprovação para experimentação do álcool $(72,7 \%)$, da maconha $(45,5 \%)$, de anfetaminas $(9,1 \%)$ e alucinógenos $(36,4 \%)$.

A questão do risco da experimentação e o abuso de substâncias psicoativas em homossexuais e bissexuais é uma temática frequentemente apontada por pesquisadores, que afirmam notar taxas de abuso e dependência de substâncias psicoativas nessa população [28]. Estudo realizado no Ceará revelou que $59,4 \%$ desta população afirma utilizar substâncias lícitas, destes, $70,9 \%$ utilizam álcool, e 22,7\% utilizam o tabaco. Para as substâncias ilícitas, o consumo foi afirmado em $40,5 \%$ destas pessoas [29].

Quando analisado o comportamento de aprovação para experimentação de substâncias e a relação com a cor da pele, notou-se que o comportamento da população negra, quanto as substâncias lícitas, foi de maior índice de aprovação para experimentação do álcool para 76,9\% dos entrevistados, e das substâncias ilícitas, o maior índice de aprovação para experimentação da maconha com $30,8 \%$. A população de cor de pele branca foi a única população que aprovou a experimentação de todas as substâncias, e a que mais aprovou a experimentação do tabaco $(32,0 \%)$, de anfetaminas $(5,0 \%)$, de inalantes $(5,0 \%)$, de hipnóticos $(13,6 \%)$, e de opiáceos $(6,8 \%)$. O índice de aprovação para experimentação de alucinógenos foi maior na população parda $(9,7 \%)$.

Em relação à idade dos entrevistados, observa-se que na faixa etária de 17 a 34 anos existe a aprovação para experimentação de todas as substâncias. Ainda, observa-se que o álcool mantém o maior índice de aprovação para experimentação em todas as faixas etárias dos participantes deste estudo.

O envolvimento, na idade da adolescência, com drogas lícitas é real, fato semelhante ao de pesquisa realizada com 210 adolescentes, na qual 6,6\% já haviam experimentado o tabaco, e $20 \%$ já haviam experimentado o álcool [30].

O álcool, no Brasil, é utilizado abusivamente por adolescentes e adultos jovens, fato que constitui importante problema de saúde pública, proporcionando sérios danos à sociedade, inclusive perfazendo a maior causa de morte nesta faixa etária [15].

Este comportamento pode estar associado a vários fatores de risco, fatos que podem levar a maior utilização e dependência dessas substâncias, e, ainda, podem levar a experimentação e envolvimento com substâncias ilícitas, que geralmente ocorre na faixa etária da adolescência e adulta jovem [17]. 
Ainda, é importante ressaltar que a faixa etária entre 23 e 28 anos de idade é a que mais aprova a experimentação das substâncias lícitas (tabaco 31,8\%, álcool 73,9\%), além do crack $(8,0 \%)$, inalantes $(8,0 \%)$, opiáceos $(9,1 \%)$. A faixa etária entre 29 e 34 anos de idade apresentou maior índice de aprovação de experimentação para anfetaminas $(7,7 \%)$ e alucinógenos (11,6\%). Para a maconha, a faixa etária de maior aprovação foi dos 35 aos 40 anos de idade, com 22,2\%.

A idade jovem se constitui o maior risco para envolvimento com substâncias, contudo, neste estudo, a maior incidência de aprovação para experimentação da maconha se apresentou na faixa etária entre 35 e 40 anos de idade, o que difere de pesquisas que revelam a diminuição do envolvimento com o decorrer da idade [26].

Em relação ao curso de graduação no qual o aluno está vinculado, observa-se a escassez de estudos que tratem dessa temática em alunos que não sejam da área da saúde, o que é apontado como necessário, tendo em vista a verificação deste comportamento em estudantes de todos os cursos pesquisados.

Pesquisa realizada em Volta Redonda/RJ aponta consumo de álcool por estudantes de medicina em 81,1\%, e 87,1\% em Salvador/BA, 83,1\% em São Paulo/SP, 85,2\% em Belo Horizonte/MG, 86,8\% em cidade da região centro-oeste, próximo ao resultado apresentado neste estudo. Aos alunos de Enfermagem, o índice de envolvimento chegou a 90,6\% em estudo realizado no nordeste brasileiro, superior aos $69,7 \%$ encontrados neste estudo [31-33].

Observa-se um comportamento de envolvimento com álcool, o maior índice de aprovação de experimentação encontrado neste estudo, como uma prática consensual nos universitários brasileiros.

O envolvimento de estudantes universitários com álcool e outras drogas é real, intenso e frequente, fato este exposto em estudo em que quase $49 \%$ dos universitários pesquisados, pelo menos uma vez na vida, já haviam experimentado alguma droga ilícita, e $80 \%$ dos que se declarara menos de 18 anos de idade afirmaram já ter consumido algum tipo de bebida alcóolica [1].

O primeiro uso da substância abriga diferenças individuais, que poderão determinar a repetição do uso desta mesma substância. Para que a repetição ocorra, será necessário o desenvolvimento da sensibilização e aprendizado na autoadministração desta substância. Esse processo de aprendizado, por sua vez, desenvolverá a dependência à substância respectiva, compreendendo as interações com as drogas e com os ambientes relacionados a ela [9].

Ainda, com a experimentação de uma determinada substância psicoativa, e consequente contato com os efeitos produzidos, poderá ser desenvolvida a fissura no indivíduo, que se refere a um intenso desejo, busca e repetição de pensamentos relacionados à substância experimentada [9].

Esse comportamento poderá incidir em risco maior à dependência, uma vez que a necessidade dos efeitos produzidos pelas substâncias está em nível quase que descontrolado pelo indivíduo.

As limitações do estudo são consequência de recorte metodológico de dada realidade e não do todo, no sentido de melhor compreendê-lo em cada região brasileira, porém é importante considerar o fato exposto, e incentivar a realização de outras pesquisas que objetivem compreender a intencionalidade e aprovação para o envolvimento com substâncias.

Dessa forma, recomenda-se a realização de mais estudo acerca da temática, que apresentem diferentes realidades e forneçam contribuições para a prevenção e identificação precoce do envolvimento com substâncias psicoativas, a fim de não haver ocorrência de dependência dessas substâncias pela população universitária.

Os fatores relacionados ao primeiro contato com as drogas são variados, dessa forma, a vivência de um conjunto de fatores de risco para o contato com as drogas, atrelado ao contexto no qual está inserido, predispõe o adolescente e o jovem à utilização de drogas, visto a interdependência desses fatores.

É possível observar que ainda se admite a possibilidade da experimentação de álcool e outras substâncias, mesmo com o desenvolvimento da tolerância a estas substâncias pelo organismo, fato que aumenta o risco da dependência das substâncias e seus efeitos. $E$, ainda, é importante ressaltar que o ambiente universitário poderia ser um espaço para construção educativa preventiva relacionada a essa temática, contudo observa-se a busca por experiências não sendo levadas em consideração as possíveis consequências.

Dessa forma, a necessidade de investimento em políticas públicas educacionais, também a nível local, que objetivem o conhecimento e a prevenção do primeiro contato com 
essas substâncias é clara, quando é considerado o cenário real de contato com essas substâncias na população universitária brasileira. Além desta população, as ações de educação em saúde devem contemplar as famílias e as comunidades locais, pois, na maioria das vezes, a primeira exposição a qualquer tipo de droga está relacionada com as influências.

Concomitantemente, a questão da experimentação e do uso do álcool e outras substâncias por universitários precisam ser debatidos nos espaços acadêmicos locais, elaborando políticas internas por parte das instituições, por conta do risco do estudante desenvolver prejuízos, que são muitos, fatos que irão interferir na sua vida, não somente no período de graduação, mas por toda a sua vida.

\section{Referências}

1. Cerutti F, Argimon IIL. Relacionamento pais e filhos e as implicações no uso de substâncias psicoativas: uma revisão sistemática. Perspect Psicol (Mar del Plata) 2015;12(1):57-65. Disponível em:

http://www.seadpsi.com.ar/revistas/index.php/pep/article/view/203/pdf

2. World Health Organization. Global status report on alcohol and health 2014. Genebra: WHO; 2014.

3. Brasil. Presidência da República. Secretaria Nacional de Políticas sobre Drogas. I Levantamento Nacional sobre o Uso de Álcool, Tabaco e Outras Drogas em Universitários das 27 Capitais Brasileiras. Brasília: Secretaria Nacional de Políticas sobre Drogas; 2010.

4. Waston AM, Zweben JE. Prática psicoterápica eficaz dos problemas com álcool e drogas. Porto Alegre: Artmed; 2009.

5. Paiva PCP, Paiva HN, Lamounier JA, Ferreira EF, César CAS, Zarzar PM. Consumo de álcool em binge por adolescentes escolares de 12 anos de idade e sua associação com sexo, condição socioeconômica e consumo de álcool por melhores amigos e familiares. Ciênc Saúde Colet 2015;20(11):3427-35. https://doi.org/10.1590/1413812320152011.18792014

6. Brasil. Ministério da Saúde. Conselho Nacional de Saúde. Resolução no 466 de 12 de dezembro de 2012. Diário Oficial da União; 13 jun 2013.

7. Polcin DL, Korcha RA, Bond JC. Interaction of motivation and psychiatric symptoms on substance abuse outcomes in sober living houses. Subst Use Misuse 2015;50(2):195204. https://doi.org/10.3109/10826084.2014.962055

8. Feldman RS. Introdução à Psicologia. 10ª ed. Porto Alegre: AMGH; 2015.

9. Organização Mundial da Saúde. Neurociência do uso e da dependência de substâncias psicoativas. São Paulo: Roca; 2006.

10. Duarte CE, Morihisa RS. Experimentação, uso, abuso e dependência de drogas. In: SENAD. Prevenção do uso de álcool e outras drogas no ambiente de trabalho Conhecer para ajudar. 3aㅡ ed. Brasília: Ministério da Justiça; 2012.

11. Silva DA. A autoestima e o comportamento suicida em estudantes universitários: uma revisão da literatura. Rev. Eletrônica Acervo Saúde 2019;23:e422. https://doi.org/10.25248/reas.e422.2019

12. Dázio EMR, Zago MMF, Fava SMCL. Uso de álcool e outras drogas entre universitários do sexo masculino e seus significados. Rev Esc Enferm USP 2016;50(5):786-92. https://doi.org/10.1590/S0080-623420160000600011

13. Araujo CM, Vieira CX, Mascarenhas CHM. Prevalência do consumo de drogas lícitas e ilícitas por estudantes universitários. Rev Eletrônica Saúde Mental Álcool Drog 2018;14(3):144-50. https://doi.org/10.11606/issn.1806-6976.smad.2018.000342

14. Brasil. Ministério da Saúde. Instituto Nacional de Câncer. Relatório de OMS sobre a epidemia Global de Tabagismo, 2008. [citado 2017 Jan 20]. Disponível em: http://www.inca.gov.br/tabagismo/ publicacoes/OMS Relatorio.pdf

15. Pinheiro BO, Andrade ALM, Micheli DD. Relação entre os níveis de atividade física e qualidade de vida no uso de drogas em adolescentes. SMAD, Rev Eletrônica Saúde Mental Álcool Drog 2016;12(3):178-87. https://doi.org/10.11606/issn.18066976.v12i3p178-187

16. Elicker E, Palazzo LS, Aerts DRGC, Alves GG, Câmara S. Uso de álcool, tabaco e outras drogas por adolescentes escolares de Porto Velho-RO, Brasil. Epidemiol Serv Saúde 2015;24(3):399-410. https://doi.org/10.5123/S1679-49742015000300006 
17. Abreu AMM, Parreira PMSD, Souza MHN, Barroso TMMDA. Perfil do consumo de substâncias psicoativas e sua relação com as características sociodemográficas: uma contribuição para intervenção breve na atenção primária à saúde. Texto Contexto Enferm 2016;25(4):e1450015. https://doi.org/10.1590/0104-07072016001450015

18. Romera LA, Martins RA, Freitas HH, Tinoco DS, Rondina RC. Tempo livre e uso de álcool e outras drogas: estudo comparativo entre estudantes universitários do Brasil e Portugal. Movimento 2018;24(3):765-76. https://doi.org/10.22456/1982-8918.81951

19. Zeferino MT, Hamilton H, Brands B, Wright MGM, Cumsille F, Khenti A. Consumo de drogas entre estudantes universitários: família, espiritualidade e entretenimento moderando a influência dos pares. Texto Contexto Enferm 2015;24(Esp):125-35. https://doi.org/10.1590/0104-07072015001150014

20. Souza J, Ornella KP, Almeida LY, Domingos SGA, Andrade LS, Zanetti ACG. Consumo de drogas e conhecimento sobre suas consequências entre estudantes de graduação em Enfermagem. Texto Contexto 2018;27(2):e5540016. https://doi.org/10.1590/0104070720180005540016

21. Brasil. Presidência da República. Secretaria Nacional de Políticas sobre Drogas. Relatório brasileiro sobre drogas. Brasília: SENAD; 2009.

22. Lermen HS, Dartora T, Capra-Ramos C. Drug addiction in prison: questions about a project of drug detoxification to incarcerated people. Estud Pesqui Psicol 2014;14(2):539-59. Disponível em: http://pepsic.bvsalud.org/pdf/epp/v14n2/v14n2a09.pdf

23. Vargas AFM, Campos MM. A trajetória das políticas de saúde mental e de álcool e outras drogas no século XX. Ciênc Saúde Colet 2019;24(3):1041-50. https://doi.org/10.1590/1413-81232018243.34492016

24. Batista CB, Vasconcelos MPN, Vecchia MD, Queiroz IS. Permanent education on harm reduction: the experience of Psychosocial Care Course in Alcohol and other Drugs. Interface (Botucatu) 2019;23:e180071. https://doi.org/10.1590/interface.180071

25. Corradi-Webster CM, Gherardi-Donato ECS. Factors associated with problematic drug use among psychiatric outpatients. Rev Latinoam Enferm 2016;24:e2815. https://doi.org/10.1590/1518-8345.1444.2815

26. Landim-Almeida CAP, Rodrigues HBP, Magalhães JM, Fernandes MA. Fatores associados à opinião favorável (ou contrária) à liberação da maconha em uma amostra de docentes e discentes universitários. SMAD Rev Eletrônica Saúde Mental Álcool Drog 2016;12(1):12-21. https://doi.org/10.11606/issn.1806-6976.v12i1p12-21

27. Schneider JA, Andretta I. Habilidades sociais como fatores de risco e proteção entre homens usuários de crack. Quaderns de Psicologia. 2017;19(2):151-61. https://doi.org/10.5565/rev/qpsicologia.1394

28. Parente JS, Belém JM, Figueiredo LS, Paiva LS, Garcia CL, Albuquerque GA. Álcool, drogas e violência: implicações para a saúde de minorias sexuais. Reprod Clim 2015;30:108-14. https://doi.org/10.1016/j.recli.2015.11.002

29. Garcia DM, Filho EM, Gilio AE, Lotufo JPB, Lo DS. Estado nutricional, autopercepção do estado nutricional e experimentação de drogas lícitas em adolescentes. Rev Paul Pediatr 2015;33(3):332-9. https://doi.org/10.1016/j.rpped.2014.11.015

30. Hall WD, Patton G, Stockings E, Weier M, Lynskey M, Morley KI et al. Why young people's substance use matters for global health. Lancet Psychiatry 2016;3(3):265-79. https://doi.org/10.1016/S2215-0366(16)00013-4

31. Gomes LS, Barroso CRD, Silvestre VA, Baylão ACP, Garcia SCM, Pacheco SJB. Consumo de álcool entre estudantes de medicina do Sul Fluminense - RJ. Rev Med (São Paulo) 2018;97(3):260-6. https://doi.org/10.11606/issn.1679-9836.v97i3p260-266

32. Fernandes MA, Moita FM, Nascimento MPV, Santos JDM, Paiva MHP. Consumo de bebidas alcoólicas em estudantes de enfermagem de um centro universitário. SMAD Rev Eletrônica Saúde Mental Álcool Drog 2019;15(2):38-44. https://doi.org/10.11606/issn.1806-6976.smad.2019.000401

33. Trindade BPA, Diniz AV, Sá-Junior AR. Uso de drogas entre estudantes universitários: uma perspectiva nacional. Rev Med Saude Brasilia 2018;7(1):52-60. Disponível em: https://portalrevistas.ucb.br/index.php/rmsbr/article/view/8641/5721 